\title{
Ion Distributions at Electrified Water-Organic Interfaces: PB-PMF Calculations and Impedance Spectroscopy Measurements
}

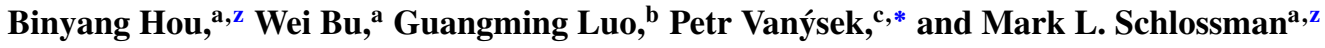 \\ ${ }^{a}$ Department of Physics, University of Illinois at Chicago, Chicago, Illinois 60607, USA \\ ${ }^{b}$ BSRF, Institute of High Energy Physics, Chinese Academy of Science, Beijing 100049, People's Republic of China \\ ${ }^{c}$ Department of Chemistry and Biochemistry, Northern Illinois University, DeKalb, Illinois 60115, USA
}

\begin{abstract}
The interface between two immiscible electrolyte solutions consisting of alkali chlorides in water and the organic electrolyte BTPPATPFB in 1,2-dichloroethane is characterized with X-ray reflectivity, interfacial tension and impedance spectroscopy measurements over a range of applied voltage between the bulk solutions. X-ray reflectivity probes the interfacial ion distribution on the sub-nanometer length scale, whereas interfacial tension and impedance spectroscopy characterize quantities such as interfacial excess charge and differential capacitance that represent integrations over the interfacial ion distribution. Predictions of interfacial ion distributions by the recently introduced PB-PMF method, which combines Poisson's equation with ion potentials of mean force, provide excellent agreement, within one to two experimental standard deviations, with both X-ray reflectivity and interfacial tension measurements. However, the agreement with the differential capacitance measured by impedance spectroscopy, and modeled by the Randles equivalent circuit, is not as good. Values of measured and calculated differential capacitance can deviate by as much as $20 \%$ for applied electric potential differences larger than approximately $\pm 100 \mathrm{mV}$. These comparisons indicate that our understanding of the ion distributions that underlie these measurements is adequate, but that further understanding of the modeling of impedance spectroscopy data is required for quantitative agreement at larger applied electric potential differences.

(C) The Author(s) 2015. Published by ECS. This is an open access article distributed under the terms of the Creative Commons Attribution 4.0 License (CC BY, http://creativecommons.org/licenses/by/4.0/), which permits unrestricted reuse of the work in any medium, provided the original work is properly cited. [DOI: 10.1149/2.0621512jes] All rights reserved.
\end{abstract}

Manuscript submitted August 4, 2015; revised manuscript received September 2, 2015. Published September 15, 2015.

Ion distributions at interfaces underlie many electrochemical and biological processes, including electron and ion transfer across biomembranes and liquid interfaces, phase transfer catalysis and solvent extraction. The interface between two immiscible electrolyte solutions (ITIES) has been used as a model system to study ion and electron transfer across liquid-liquid interfaces by electrochemical techniques. $^{1,2}$ These techniques characterize the interfacial ion distribution in terms of integrated properties such as interfacial excess charge or differential capacitance, which can be calculated by integrating the ion distribution over the spatial coordinate perpendicular to the interface.

The differential capacitance of ITIES has been widely investigated. $^{3-5}$ Impedance spectroscopy data for ITIES are often modeled by the Randles equivalent circuit to yield the interfacial differential capacitance. ${ }^{6}$ Although this model is convenient in many circumstances, its limitations have been discussed in the literature. For example, Samec and co-authors reported limited agreement between the results from interfacial tension and impedance spectroscopy measurements of the interface between aqueous and 1,2dichloroethane (DCE) electrolyte solutions and suggested that the Randles equivalent circuit might be at fault at high electric potential differences. 7,8

Impedance spectroscopy studies of ITIES have measured interfacial differential capacitance that depends on the nature of the ions. ${ }^{4,9-14}$ Interfacial differential capacitance has been used to test theories of interfacial ion distributions; however, the most popular theories are based upon the Gouy-Chapman theory, ${ }^{15,16}$ which does not account for differences between ions and often fails to explain the variation of the shape and magnitude of differential capacitance with electric potential difference..$^{9,13,14,17}$ Only partial success has been achieved by the introduction of other models for the differential capacitance of liquid-liquid interfaces. ${ }^{3,9-12}$ Recent theoretical investigations have utilized the Poisson-Nernst-Planck model to interpret the Randles equivalent circuit in modeling impedance spectroscopy, ${ }^{18-20}$ though this approach also ignores the role of specific ion-solvent interactions and the structure of the liquid-liquid interface. Several authors have proposed substantial modifications to the interfacial structure. For example, Schmickler et al. used a mixed boundary layer to consider the

*Electrochemical Society Fellow.

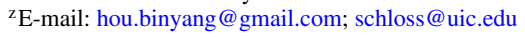

effect of overlapping ion distributions from each phase. ${ }^{13,14,21}$ Daikhin and co-authors modeled ion penetration across the interface by using a free energy profile of ion transfer that varied smoothly through the interface. $^{22,23}$ In spite of many studies of ITIES that utilized impedance spectroscopy and interfacial tension measurements, these techniques did not lead to a detailed understanding of the interfacial ion distribution.

Recent X-ray reflectivity measurements that probe the ion distribution, that is, the variation of ion concentration with distance from the interface, on the sub-nanometer length scale have further demonstrated the inadequacies of Gouy-Chapman theory. ${ }^{24-28}$ Motivated by the free energy model of Daikhin and co-workers, ${ }^{22,23}$ an ion-specific Poisson equation, which incorporated a potential of mean force (PMF) for each ion, produced excellent agreement with X-ray reflectivity measurements. ${ }^{24-27}$ This theory, referred to as the PB-PMF theory, accounts for interactions and correlations between ions and solvents that are left out of Gouy-Chapman theory. PB-PMF theory was used to explain the condensation of monovalent organic anions at the electrified interface between electrolyte solutions of water and 1, 2-dichloroethane. ${ }^{27}$ More recent X-ray reflectivity studies of liquidliquid interfaces between a series of aqueous alkali chloride solutions and 1,2-dichloroethane electrolyte solutions of BTPPATPFB showed that ion distributions from PB-PMF calculations are in excellent agreement with the results of both $\mathrm{X}$-ray reflectivity and interfacial tension measurements. ${ }^{26}$

In this study, the results of impedance spectroscopy measurements on interfaces between two electrolyte solutions are compared to X-ray reflectivity and interfacial tension measurements. Although PB-PMF theory provides a consistent explanation of the nanoscale ion distribution probed by X-ray reflectivity and the interfacial excess surface charge measured by interfacial tension, its predictions do not agree with the differential capacitance derived from a Randles equivalent circuit analysis of impedance spectroscopy. The differential capacitance is observed to be asymmetric about its minimum and deviations with the PB-PMF theory occur on either the plus or minus side of the minimum, depending upon the system, for applied electric potentials larger than approximately $100 \mathrm{mV}$. Since differential capacitance is just a measure of the ion distribution that has been described adequately by the PB-PMF theory in the context of X-ray reflectivity and interfacial tension measurements, these results suggest that our knowledge of the ion distributions is adequate, but that further understanding of the modeling of impedance spectroscopy data is required. 


\section{Experimental Methods and Materials}

Liquid-liquid interfaces between $10 \mathrm{mM}$ aqueous solutions of alkali chlorides and a $5 \mathrm{mM} \mathrm{1,} \mathrm{2-dichloroethane}$ (DCE) solution of bis(triphenyl phosphoranylidene) ammonium tetrakis(pentafluorophenyl) borate $\left(\mathrm{BTPPA}^{+}, \mathrm{TPFB}^{-}\right.$) were studied. The galvanic cell is represented by the scheme: $\mathrm{Ag}|\mathrm{AgCl}| 10 \mathrm{mM}$ $\mathrm{XCl}$ (water) || $5 \mathrm{mM} \mathrm{BTPPATPFB}$ (DCE) $\mid 10 \mathrm{mM} \mathrm{LiCl}+1 \mathrm{mM} \mathrm{BTP}-$ $\mathrm{PACl}$ (water) $|\mathrm{AgCl}| \mathrm{Ag}$, where $\mathrm{Ag} \mid \mathrm{AgCl}$ represents an electrode of $\mathrm{Ag}$ wire coated with $\mathrm{AgCl}, \mathrm{X}$ is the alkali ion $\mathrm{Li}^{+}, \mathrm{Na}^{+}, \mathrm{Rb}^{+}$or $\mathrm{Cs}^{+}$, and || represents the liquid-liquid interface of interest. Note that $\sim 54 \%$ of BTPPATPFB is dissociated in DCE at $5 \mathrm{mM}$ concentration at room temperature. ${ }^{28,29}$

Materials. - Alkali chloride salts $(\mathrm{NaCl}$ and $\mathrm{LiCl}$, certified ACS purchased from Fisher Scientific, $\mathrm{RbCl}$ 99.99\% and $\mathrm{CsCl}$ 99.999\% by trace metals basis purchased from Aldrich) were roasted at $500{ }^{\circ} \mathrm{C}$ for $30 \mathrm{~min}$. to remove organic impurities. Water was produced by a Nanopure UV Barnstead system. 1,2-dichloroethane (CHROMASOLV for HPLC, $\geq 99.8 \%$, from Aldrich) was purified by passing it through a column of basic alumina six times. The interfacial tension between pure water and DCE, measured to be $28.2 \pm 0.2 \mathrm{mN} / \mathrm{m}$ at $23^{\circ} \mathrm{C}$, in agreement with literature values, ${ }^{30}$ remained stable for many hours immediately after the formation of the interface. The supporting electrolyte in the organic phase, bis(triphenyl phosphoranylidene) ammonium tetrakis(pentafluorophenyl)borate, was prepared by metathesis of bis(triphenyl phosphoranylidene) ammonium chloride (BTPPACl 97\% from Aldrich) and potassium tetrakis(pentafluorophenyl)borate (KTPFB from Boulder Scientific Company) in 2: 1 mixtures of methanol and water, followed by recrystallization in distilled acetone. ${ }^{31}$ Additional information on the materials in use can be found in previous work..$^{26,32}$

Liquid-liquid sample cell.- All measurements were carried out in a four-electrode glass cell with a flat circular water-DCE interface with area of about $38 \mathrm{~cm}^{2}$ (diameter of $\sim 7 \mathrm{~cm}$ ) (Fig. 1). ${ }^{26}$ The liquid-liquid interface was pinned by the top edge of a Teflon strip and flattened by adjusting the volume of the lower DCE phase. Voltage was applied across the liquid-liquid interface using a four-electrode potentiostat (1287 Electrochemical Interface, Solartron Instruments, England). Two square platinum meshes $\left(\sim 9 \mathrm{~cm}^{2}\right.$ each) acted as counter electrodes $\left(\mathrm{CE}_{1}\right.$ and $\left.\mathrm{CE}_{2}\right)$. Two glass Luggin capillaries, located within 2 to $3 \mathrm{~mm}$ of the interface, were used with reference electrodes $\left(\mathrm{RE}_{1}\right.$ and $\left.\mathrm{RE}_{2}\right)$. The electric potential difference between the water and or-

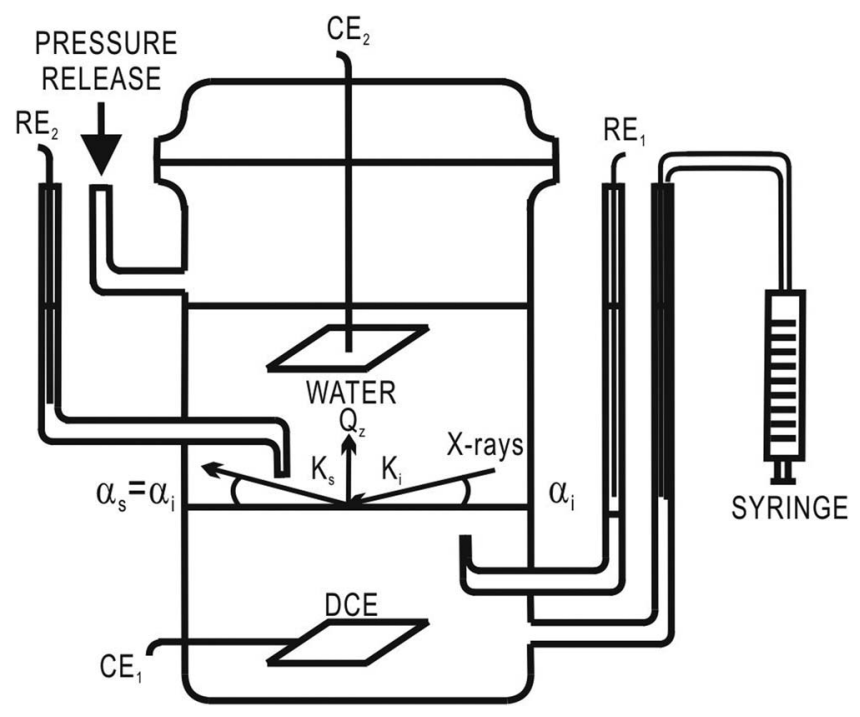

Figure 1. Circular glass sample cell and X-ray kinematics, adapted with permission (c) 2013 American Chemical Society. ${ }^{26}$ ganic (DCE) phases, $\Delta \phi^{w-o}=\Delta \phi^{\text {water }}-\Delta \phi^{\text {organic }}$, is the difference between the applied electrochemical cell potential measured by the potentiostat and the potential of zero charge determined by interfacial tension measurements $\left(\Delta \phi^{w-o}=\Delta \phi_{c e l l}^{w-o}-\Delta \phi_{p z c}^{w-o}\right)$. The polarization window revealed by cyclic voltammetry determined the experimental range of $\Delta \phi^{w-o}$.

Interfacial tension measurements. - Interfacial tension was measured with a Cahn microbalance, which measures the weight of a Teflon Wilhelmy plate fully submerged in the top (water) phase. The bottom edge of the plate was placed in contact with the liquid-liquid interface. This method was used previously ${ }^{33-35}$ to obtain interfacial tension values in excellent agreement with literature measurements that used the ring, pendant drop, and maximum bubble pressure methods. ${ }^{36-38}$

Cyclic voltammetry and impedance spectroscopy measurements.Cyclic voltammograms were measured at a scan rate of $5 \mathrm{mV} \mathrm{s}^{-1}$ using the Solartron potentiostat. Impedance spectroscopy was measured with the addition of a frequency response analyzer (1255 FRA, Solartron Instruments, England). A $5 \mathrm{mV}$ rms AC voltage was applied over the frequency range $0.2 \mathrm{~Hz}-10 \mathrm{kHz}$. After changing the applied voltage, but prior to any measurement, the samples were allowed to relax for about $10 \mathrm{~min}$. at the extreme potentials at the ends of the potential window or for $5 \mathrm{~min}$. at all other potentials to allow the system to reach steady state. In this state the total current through the electrochemical cell is on the order of $1 \mu \mathrm{A}$ (i.e., $0.026 \mu \mathrm{A} \mathrm{cm}^{-2}$ ), except at the most negative and positive potentials for which the cell exhibits a maximum total current of $8 \mu \mathrm{A}$ (i.e., $0.2 \mu \mathrm{A} \mathrm{cm}{ }^{-2}$ ). After measurement at each potential, the cell potential was ramped back to the open circuit potential to relax for about $5 \mathrm{~min}$. and then ramped to the next potential at a speed of no more than $1 \mathrm{mV} \mathrm{s}^{-1}$. Measurements at the largest positive and negative electric potential differences were taken after the majority of measurements at lower electric potential differences were completed because a relatively larger amount of inorganic ions would be transferred to the DCE phase and a longer relaxation time would then be required for them to partition back to the aqueous phase at the open circuit potential. The same potential cycling method was used for X-ray reflectivity and interfacial tension measurements. ${ }^{26}$

$X$-ray reflectivity measurements. - X-ray reflectivity measurements were carried out at the ChemMatCARS beamline 15-ID at the Advanced Photon Source (Argonne National Laboratory, USA) with a liquid surface instrument and measurement techniques described in detail elsewhere. ${ }^{32,39}$ The reflectivity data were measured as a function of the wave vector transfer perpendicular to the interface, $Q_{z}=\vec{k}_{s}-\vec{k}_{i}=(4 \pi / \lambda) \sin \alpha_{i}$, where $\vec{k}_{i}$ and $\vec{k}_{s}$ are the incident and reflected wave vectors shown in Fig. $1, \lambda=0.41255 \pm 0.00005 \AA$ is the X-ray wavelength and the angle of reflection $\alpha_{s}$ in Fig. 1 is equal to the angle of incidence $\alpha_{i}$. The in-plane components of the wave vector transfer are given by $Q_{x}=Q_{y}=0$.

\section{Results and Discussion}

Cyclic voltammetry. - Figure 2 shows the first five cycles of a cyclic voltammogram (CV) measured at a scan rate of $5 \mathrm{mV} \mathrm{s}^{-1}$ from the sample containing $10 \mathrm{mM} \mathrm{NaCl}$ in water and $5 \mathrm{mM} \mathrm{BTPPATPFB}$ in DCE. Cyclic voltammograms for samples with $\mathrm{Li}, \mathrm{Rb}$, or $\mathrm{Cs}$ replacing $\mathrm{Na}$ are similar in shape, but have different range in electric potential difference $\Delta \phi^{w-o}$ because of different Gibbs energies of transfer for the ions. ${ }^{26}$ The smooth I-V curve confirms that the interface is clean without noticeable electrochemically active species.

Interfacial tension measurement.- Interfacial tension data for $\mathrm{NaCl}$ is shown in Fig. 3 and published elsewhere are samples with $\mathrm{LiCl}, \mathrm{RbCl}$, and $\mathrm{CsCl}^{26}$ Hyperbolic cosine fits to the interfacial tension data determine the potential of zero charge $\Delta \phi_{p z c}^{w-o}$, which 


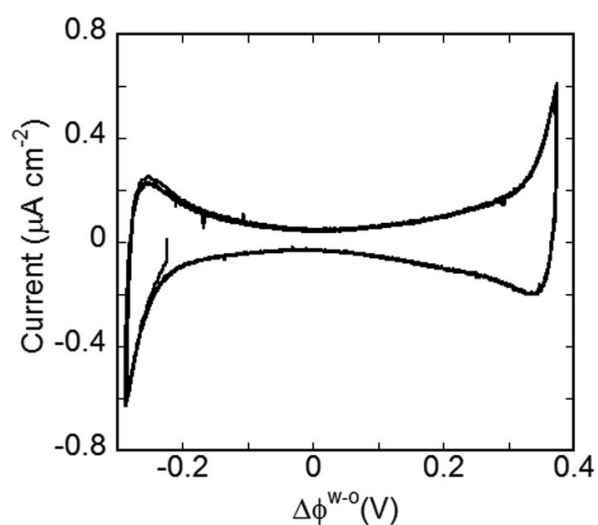

Figure 2. Cyclic voltammogram for the interface between $10 \mathrm{mM} \mathrm{NaCl}$ (water) and $5 \mathrm{mM}$ BTPPATPFB (DCE) measured at a scanning rate of $5 \mathrm{mV} \mathrm{s}^{-1}$ for the illustrated range of electric potential difference $\Delta \phi^{w-o}$. The first five cycles are shown.

occurs at the apex of the fitted curve. The fitted values of $\Delta \phi_{p z c}^{w-o}$ are $0.365 \pm 0.003 \mathrm{~V}$ for the $\mathrm{LiCl}$ sample, $0.374 \pm 0.008 \mathrm{~V}$ for $\mathrm{NaCl}, 0.360$ $\pm 0.006 \mathrm{~V}$ for $\mathrm{RbCl}$, and $0.380 \pm 0.007 \mathrm{~V}$ for $\mathrm{CsCl}$, respectively. ${ }^{26}$ The line shown in Fig. 3 is a spline fit to the data that will be used subsequently to calculate the excess surface charge described later.

$X$-ray reflectivity data and ion distribution analysis. - X-ray reflectivity measurements and analysis for four samples with different alkali chlorides were published previously. ${ }^{26,32}$ We review the data and analysis for the $\mathrm{NaCl}$ sample and highlight details relevant to this work. Figure 4 illustrates $\mathrm{X}$-ray reflectivity data $R\left(Q_{z}\right)$ from the interface between a $10 \mathrm{mM} \mathrm{NaCl}$ aqueous solution and a $5 \mathrm{mM}$ DCE solution of BTPPATPFB normalized to the Fresnel reflectivity $R_{F}\left(Q_{z}\right)$ calculated for a flat, structureless interface. ${ }^{39}$ Increasing the potential above $\Delta \phi^{w-o}=0$ leads to the development of a peak in the reflectivity at $Q_{z} \approx 0.13 \AA^{-1}$, while only small changes are observed for $\Delta \phi^{w-o}<0$.

$\mathrm{X}$-ray reflectivity measurements probe the gradient of the electron density profile in the direction perpendicular to the interface, $d \rho(z) / d z$, with sub-nanometer spatial resolution. The variation of ion concentration with $z$ leads to an electron density profile. In analogy to interference fringes generated by reflections from the top and bottom of thin films, interfaces that consist of ordered molecular layers produce Kiessig fringes in the variation of reflectivity with $Q_{z}$. The peaks in the reflectivity data in Figure 4 are essentially the first peak, or Kiessig fringe, in an interference pattern produced by a layer of

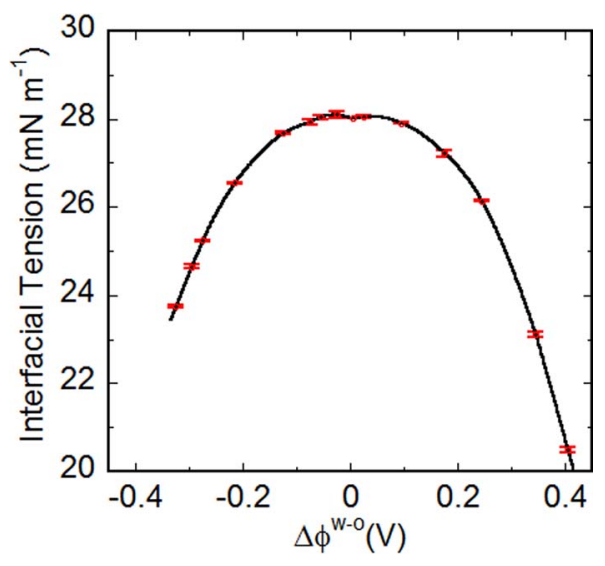

Figure 3. (Color online) Electrocapillary curve for interface between $10 \mathrm{mM}$ $\mathrm{NaCl}$ in water and $5 \mathrm{mM}$ BTPPATPFB (DCE). Data are shown by symbols; the line is a spline fit to the data.

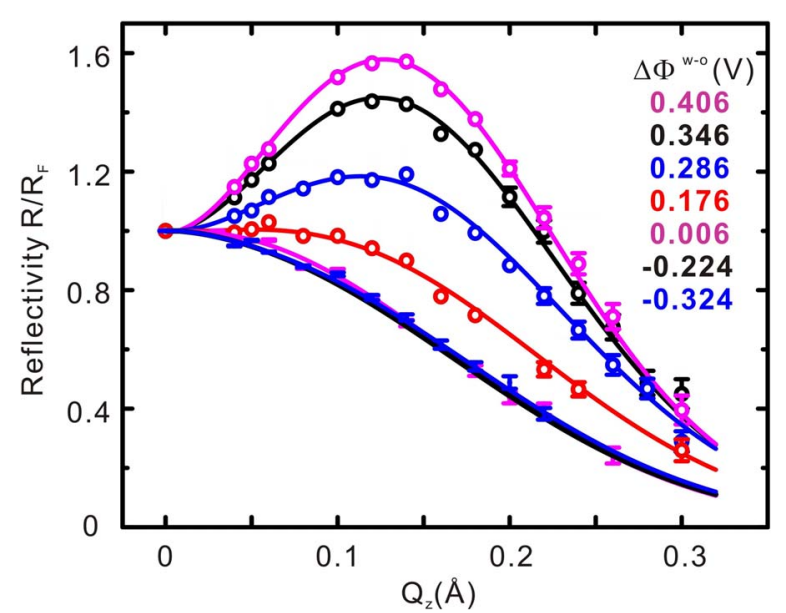

Figure 4. (Color on-line) X-ray reflectivity normalized to Fresnel reflectivity, $R\left(Q_{z}\right) / R_{F}\left(Q_{z}\right)$, for various electric potential differences $\Delta \phi^{w-o}$ as a function of wave vector transfer $Q_{z}$ from the interface between a $10 \mathrm{mM} \mathrm{NaCl}$ aqueous solution and a $5 \mathrm{mM}$ DCE solution of BTPPATPFB. Curves are ordered from top to bottom according to decreasing $\Delta \phi^{w-o}$. The data for $\Delta \phi^{w-o}=$ $0.006 \mathrm{~V},-0.224 \mathrm{~V}$, and $-0.324 \mathrm{~V}$ nearly overlap.

ions at the interface. Additional peaks might have been observed if the X-ray reflectivity could have been measured to larger values of $Q_{z}$. However, measurements to smaller values of $R\left(Q_{z}\right)$ that occur at larger $Q_{z}$ are prohibitively time-consuming. The data shown in Figure 4 are the result of measurements that span eight orders of magnitude, from $R\left(Q_{z}\right)=1$ to $R\left(Q_{z}\right) \approx 10^{-8}$. The data have been normalized by the Fresnel reflectivity $R_{F}\left(Q_{z}\right)$ that was calculated for an ideal interface between the bulk aqueous solution and the bulk organic solution for which the electron density change is a step function at the interface. ${ }^{39}$

At positive $\Delta \phi^{w-o}$, the interfacial concentration of $\mathrm{TPFB}^{-}$and $\mathrm{Na}^{+}$are enhanced over the bulk values, while those of BTPPA ${ }^{+}$and $\mathrm{Cl}^{-}$are depleted. In this case, the TPFB ${ }^{-}$ion provides the dominant contribution to the electron density contrast at the interface, largely as a result of its twenty fluorine atoms $\left(\rho_{T P F B^{-}}=0.628 \mathrm{e}^{-} \AA^{-3}\right.$ compared to $\left.\rho_{D C E}=0.381 \mathrm{e}^{-} \AA^{-3}\right)$. The peaks in Figure 4 at positive $\Delta \phi^{w-o}$ signal the formation of an interfacial layer of $\mathrm{TPFB}^{-}$ions. The increase of the peak intensity with $\Delta \phi^{w-o}$ indicates the progressive increase of interfacial $\mathrm{TPFB}^{-}$concentration within this layer. The absence of peaks for negative $\Delta \phi^{w-o}$ is consistent with the electron density contributions of $\mathrm{BTPPA}^{+}$and $\mathrm{Cl}^{-}$ions despite the expected increase of their interfacial concentrations. ${ }^{26}$ In this case $\left(\Delta \phi^{w-o}<0\right)$ the large electron density of $\mathrm{TPFB}^{-}$ions is irrelevant since they are depleted from the interface along with $\mathrm{Na}^{+}$ions.

The potential of mean force describes the distribution of ions near a planar interface, ${ }^{40}$

$$
c_{i}(z)=c_{i}^{o} \exp \left[-\left(w_{i}(z)-w_{i}^{o}\right) / k_{B} T\right],
$$

where $i$ represents different species of ions, $c_{i}^{o}$ is the bulk concentration of ion $i$ calculated from the Nernst equation, ${ }^{41} w_{i}(z)$ is the $z$-dependent potential of mean force (PMF) for each ion $i,{ }^{22,24-27,32,42} w_{i}^{o}$ is the constant potential of mean force for ion $i$ in the bulk liquid, and $k_{B} T$ is Boltzmann's constant times the temperature. Equation 1 is an exact expression under the condition that the ion distribution is independent of the in-plane $x-y$ coordinates. Poisson's equation can then be written as

$$
\frac{d}{d z}\left(\varepsilon_{0} \varepsilon_{r}(z) \frac{d}{d z} \phi(z)\right)=-\sum_{i} Z_{i} e c_{i}^{o} \exp \left[-\left(w_{i}(z)-w_{i}^{o}\right) / k_{B} T\right],
$$

where $\varepsilon_{0}$ is the permittivity of free space, $\varepsilon_{r}(z)$ describes the step function variation of the relative permittivity from the value for DCE $(10.43)^{43}$ for $z<0$ to that of water (78.45) for $z>0, \phi(z)$ is the 
electric potential, $Z_{i} e$ is the charge of ion $i$, where $e$ is the elementary unit of charge and $Z_{i}= \pm 1$ for monovalent cations and anions. The spatial dependence of $\varepsilon_{r}(z)$ is ignored in this study, which is partially justified by previous calculations that show that it has a negligible effect on the type of measurements presented here. ${ }^{25}$

The potential of mean force $w_{i}(z)$ will be written as a sum of the ion interactions due to the mean electric field that were previously described by Gouy and Chapman in the Poisson-Boltzmann equation, ${ }^{15,16}$ which we call $w_{i}^{P B}(z)$, and the remaining interactions of ion $i$ with other ions and solvent molecules, which we call $w_{i}^{n P B}(z)$ (where the superscript stands for non-Poisson-Boltzmann), ${ }^{22,24-27,32,42}$

$$
\begin{aligned}
w_{i}(z) & =w_{i}^{P B}(z)+w_{i}^{n P B}(z) \\
& =Z_{i} e \phi(z)+w_{i}^{n P B}(z)
\end{aligned}
$$

The non-Poisson-Boltzmann term accounts for such effects as the interfacial structure, the ion and solvent molecules' sizes and shapes, and spatial correlations between ions and solvent molecules. As described below, a parameterized analytic form is chosen to represent $w_{i}^{n P B}(z)$ for some of the ions. Solution of the Poisson equation (Eq. 1) with the potential of mean force given by Eq. 3 yields a parameterized ion concentration profile, which is then converted to a parameterized electron density profile whose parameters are fit to the $\mathrm{X}$-ray reflectivity data. The Poisson equation in Eq. 2 was solved numerically for the electric potential $\phi(z)$ using the quasi-linearization procedure $^{44,45}$ subject to the following constraints: (a) the measured potential difference $\Delta \phi^{w-o}$ between the bulk liquids on either side of the liquid/liquid interface, (b) electroneutrality in each bulk phase $(d \phi / d z=0$ far from the interface and the electrodes), and (c) electrostatic boundary conditions $\left(\varepsilon_{r}^{\text {water }} d \phi /\left.d z\right|_{z=0+}=\varepsilon_{r}^{D C E} d \phi /\left.d z\right|_{z=0-}\right.$ and $\phi(z=0+)=\phi(z=0-))$.

The non-Poisson-Boltzmann potentials of mean force $w_{i}^{n P B}(z)$ for $\mathrm{Na}^{+}$and $\mathrm{Cl}^{-}$were taken from molecular dynamics (MD) simulations in the literature. ${ }^{27,46}$ The values of $w_{i}^{n P B}(z)$ for $\mathrm{BTPPA}^{+}, \mathrm{Li}^{+}, \mathrm{Rb}^{+}$, and $\mathrm{Cs}^{+}$are modeled by the complementary error function, $\operatorname{erf} c(z)$, which provides a smooth, monotonic free energy profile through the interface,

$$
w_{i}^{n P B}(z)-w_{i}^{\mathrm{o}, p}=\left(w_{i}^{n P B}(0)-w_{i}^{\mathrm{o}, p}\right) \frac{\operatorname{erf} c\left[\left(|z|-\delta_{i}^{p}\right) / L_{i}^{p}\right]}{\operatorname{erfc}\left[-\delta_{i}^{p} / L_{i}^{p}\right]},
$$

where the superscript $p(=w, o)$ refers to either the water phase $(z>0)$ or the organic phase $(z<0), w_{i}^{\mathrm{o}, \text { oil }}-w_{i}^{\mathrm{o}, \text { water }}$ is the standard Gibbs energy of transfer of ion $i$ from the water to the organic phase, $\delta_{i}^{p}$ is an offset to ensure continuity of $w_{i}^{n P B}(z)$ at the interface (at $z=0$ ), and $L_{i}^{p}$ characterizes the decay of $w_{i}^{n P B}(z)$ from $w_{i}^{n P B}(0)$ to its bulk values $w_{i}^{\mathrm{o}, \text { water }}$ and $w_{i}^{\mathrm{o}, \text { oil }}$. The function $w_{i}^{n P B}(z)$ for $\mathrm{TPFB}^{-}$anions was obtained previously by fitting to the X-ray reflectivity of the $\mathrm{NaCl}$ sample measured at $\Delta \phi^{w-o}=0.346 \mathrm{~V}^{26}$ The $w_{i}^{n P B}(z)$ for $\mathrm{BTPPA}^{+}$ cations was fit to either interfacial excess charge $\mathrm{e}^{26}$ or capacitance data described later in this work. Values of $w_{i}^{n P B}(z)$ for $\mathrm{Li}^{+}, \mathrm{Rb}^{+}$and $\mathrm{Cs}^{+}$ were obtained previously by fitting to X-ray reflectivity data from $\mathrm{LiCl}, \mathrm{RbCl}$ and $\mathrm{CsCl}$ samples at one positive high potential. ${ }^{26}$

When Eq. 4 is used to model $w_{i}^{n P B}(z)$ for TPFB ${ }^{-}$, the amplitudes of the peaks in the X-ray reflectivity data are underestimated by the fits, suggesting that the predicted concentration near the interface is too low. The interfacial concentration can be increased by adding a Gaussian minimum to Eq. 4, which represents phenomenologically the effect of ion-ion correlations, ${ }^{26,27}$

$$
\begin{aligned}
w_{T P F B}^{n P B}(z)-w_{T P F B}^{\mathrm{o}, p}= & \left(w_{T P F B}^{n P B}(0)-w_{T P F B}^{\mathrm{o}, p}\right) \\
& \times \frac{\operatorname{erfc}\left[\left(|z|-\delta_{T P F B}^{p}\right) / L_{T P F B}^{p}\right]}{\operatorname{erfc}\left[-\delta_{T P F B}^{p} / L_{T P F B}^{p}\right]} \\
& +D \exp \left[-\left(z-z_{0}\right)^{2} / 2 \sigma_{P M F}^{2}\right],
\end{aligned}
$$

for $z<0$, with constant offset $z_{0}$, where $D$ and $\sigma_{P M F}$ are the amplitude and width of the Gaussian. The expression in Eq. 5 is used only for $\mathrm{TPFB}^{-}$ions. A unique potential of mean force $w_{i}^{n P B}(z)$ for each ion provides excellent agreement with $\mathrm{X}$-ray data for all samples at all measured potentials except for one extremely high positive potential for both the $\mathrm{LiCl}$ (not shown in this work) and $\mathrm{NaCl}$ samples which required a slightly different $\mathrm{PMF}$ for the $\mathrm{TPFB}^{-}$ion. ${ }^{26,32}$

$\mathrm{X}$-ray reflectivity measurements are influenced by thermal fluctuations of the interface, referred to as capillary waves, ${ }^{47}$ whose effect is included by convoluting the intrinsic electron density profile with a Gaussian function of width $\sigma$, which represents the roughening of the interface by capillary waves. ${ }^{39}$

To summarize, we fit the X-ray reflectivity data by using numerical solutions of Eq. 2, including the expression for the potential of mean force in Eq. 3, and the appropriate choice of $w_{i}^{n P B}(z)$. The ion concentration profiles calculated from PB-PMF theory are converted to an electron density profile $\rho(z)$, which is used to calculate X-ray reflectivity (by use of Parratt's algorithm ${ }^{48}$ ) that can be compared to the measured X-ray reflectivity. Values of $w_{i}^{n P B}(z)$ are determined either by an MD simulation or by a parameterized analytic form that is fit to data. The parameters for a particular ion's $w_{i}^{n P B}(z)$ are fit to only one set of data (at a chosen value of electric potential difference $\Delta \phi^{w-o}$ ). This provides a library of potentials of mean force that are then used to describe data at all other values of $\Delta \phi^{w-o}$. When all potentials of mean force for a sample are available, either from MD simulations or prior fitting of a particular set of data, then the only remaining fitting parameters are a small offset in $Q_{z}$, on the order of $5 \times 10^{-4} \AA^{-1}$, which accounts for a slight misalignment of the X-ray reflectometer instrument, and the interfacial roughness $\sigma$, whose fit values have been shown to be consistent with capillary wave theory. ${ }^{26}$ Additional details of the fitting procedure can be found in the literature. ${ }^{26,32}$

Interfacial excess charge.- The excess charge per unit area of the interface $Q_{t o t}\left(\Delta \phi^{w-o}\right)$ is determined by the variation of interfacial tension $\gamma$ with applied voltage $\Delta \phi^{w-o}, Q_{t o t}\left(\Delta \phi^{w-o}\right)=$ $-\left(\partial \gamma / \partial \Delta \phi^{w-o}\right)_{T, p, \mu_{i}}$, where $T$ is the thermodynamic temperature, $p$ is the external pressure and $\mu_{i}$ is the chemical potential of species $i^{2,49}$ Figure 5 compares the interfacial excess charge $Q_{t o t}\left(\Delta \phi^{w-o}\right)$ calculated from a spline fit of the measured interfacial tension to the interfacial excess charge determined by the PB-PMF analysis of the $\mathrm{X}$-ray reflectivity. As described, given the potential of mean force for each ion, the PB-PMF theory can predict the distributions of all ions at any value of $\Delta \phi^{w-o}, c_{i}\left(\Delta \phi^{w-o}, z\right)$, which are then integrated and

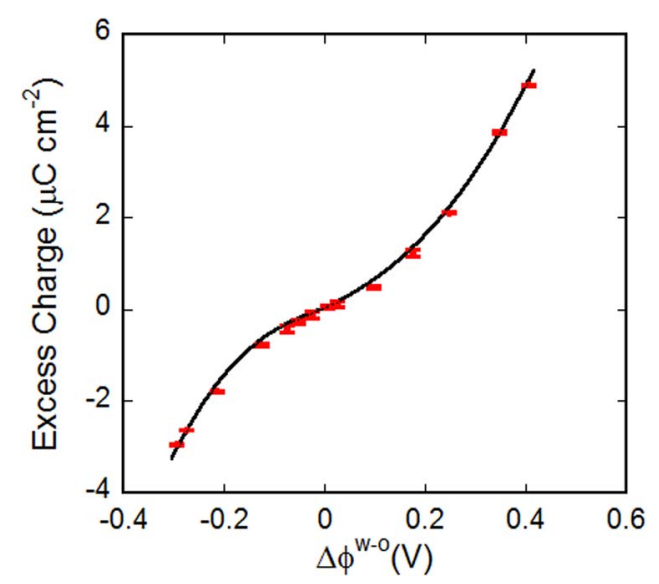

Figure 5. (color online) Interfacial excess charge $Q_{t o t}\left(\Delta \phi^{w-o}\right)$ for the interface between $10 \mathrm{mM} \mathrm{NaCl}$ (water) and $5 \mathrm{mM}$ BTPPATPFB (DCE). Data are determined from the interfacial tension measurements shown in Fig. 3; line is determined from a PB-PMF analysis described in the text. 

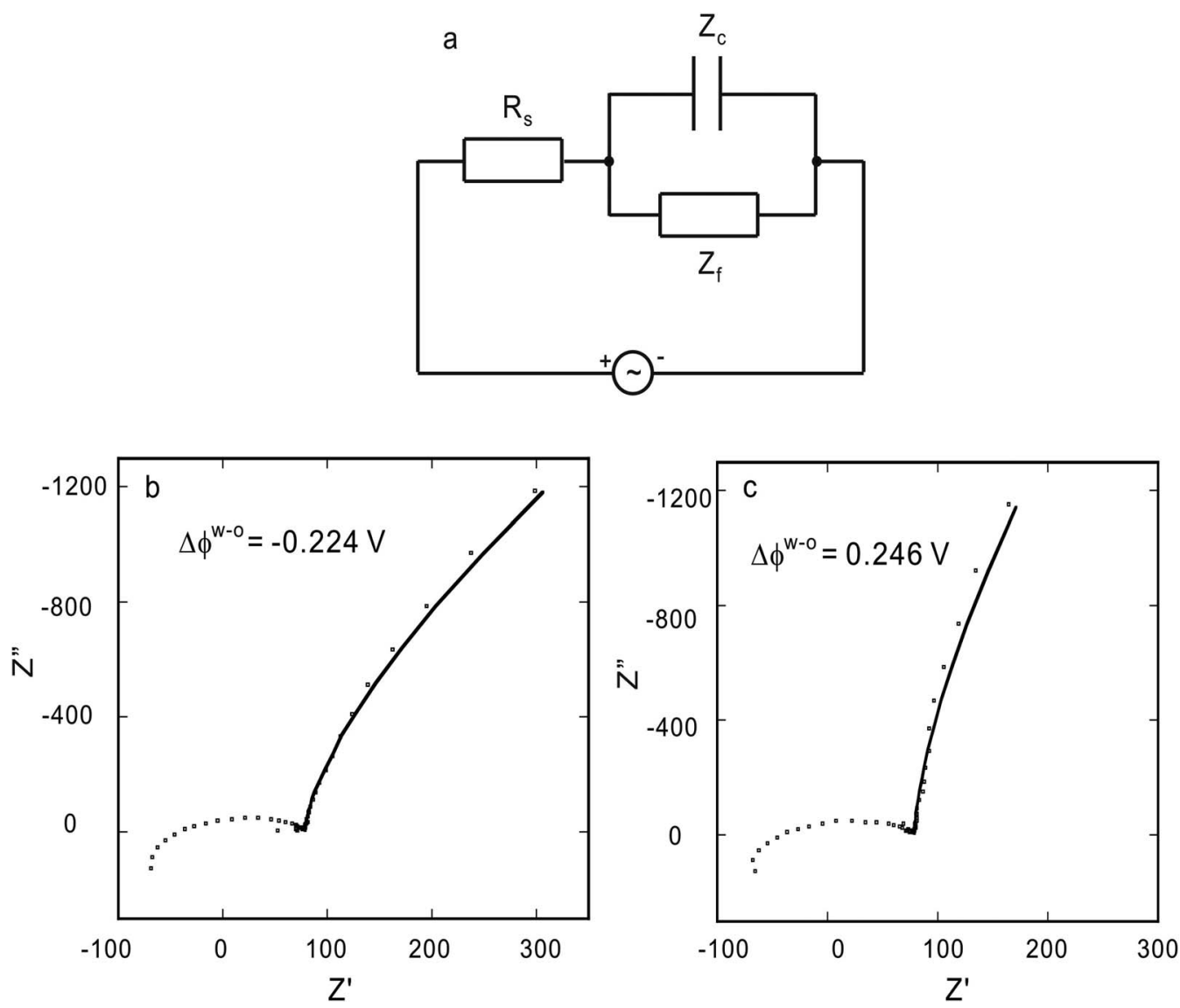

Figure 6. a) Randles equivalent circuit. b) and c) Impedance data and fits to the Randles equivalent circuit at $\Delta \phi^{w-o}=-0.224 \mathrm{~V}$ and $0.246 \mathrm{~V}$, respectively; only the lower frequencies(from 0.2 to $40 \mathrm{~Hz}$ ) were fit. The real and imaginary parts of the impedance are $Z^{\prime}$ and $Z^{\prime \prime}$ in the unit of $\Omega$.

summed to determine $Q_{t o t}\left(\Delta \phi^{w-o}\right)$,

$$
Q_{\text {tot }}\left(\Delta \phi^{w-o}\right)=\sum_{i} \int_{z=0}^{b u l k} Z_{i} e c_{i}\left(\Delta \phi^{w-o}, z\right) d z,
$$

where $Z_{i} e$ is the charge of ion $i$ in coulombs and the summation is taken over all 4 ions (i.e., the supporting electrolytes in the aqueous and organic phases). The value of $Q_{t o t}\left(\Delta \phi^{w-o}\right)$ has the same magnitude, but opposite sign depending upon which bulk phase (water or DCE) is used in the integration limits.

The PB-PMF calculation of the interfacial excess charge shown in Fig. 5 relies upon knowledge of the potentials of mean force for all four ions: $\mathrm{Na}^{+}, \mathrm{Cl}^{-}, \mathrm{BTPPA}^{+}$, and $\mathrm{TPFB}^{-}$. As stated previously, the potentials of mean force for $\mathrm{Na}^{+}$and $\mathrm{Cl}^{-}$were taken from MD simulations, ${ }^{27,46}$ the potential of mean force for $\mathrm{TPFB}^{-}$is fit to the $\mathrm{X}$ ray reflectivity data, but the $\mathrm{X}$-ray contrast for $\mathrm{BTPPA}^{+}$in $\mathrm{DCE}$ is too small to determine the BTPPA ${ }^{+}$potential of mean force precisely from the X-ray data. Instead, we used the analytic form for the potential of mean force in Eq. 4 and PB-PMF theory to determine the potential of mean force for BTPPA $^{+}$from the interfacial tension measurement of $Q_{t o t}\left(\Delta \phi^{w-o}\right)$. This fitting takes place over the range $\Delta \phi^{w-o}<0$ for which the $\mathrm{BTPPA}^{+}$concentration is enhanced at the interface. Subsequently, this BTPPA ${ }^{+}$potential of mean force was used, along with the other potentials of mean force, to fit the $\mathrm{X}$-ray reflectivity data at $\Delta \phi^{w-o}=0.346 \mathrm{~V}$ shown in Fig. 4, though the effect of $\mathrm{BTPPA}^{+}$at this positive potential difference is negligible. As shown in Fig. 5, use of these potentials of mean force and the PB-PMF theory to calculate the interfacial excess charge provides excellent agreement with the interfacial excess charge determined by interfacial tension data. Again, the effect of the $\mathrm{BTPPA}^{+}$at $\Delta \phi^{w-o}>0$ is negligible and the excellent match between the PB-PMF theory and $Q_{t o t}\left(\Delta \phi^{w-o}\right)$ from interfacial tension demonstrates the consistency of the interfacial tension with the ion distributions determined from X-ray reflectivity. A similarly high quality match was found previously when the same potentials of mean force for $\mathrm{Cl}^{-}, \mathrm{BTPPA}^{+}$, and $\mathrm{TPFB}^{-}$were used to calculate $Q_{t o t}\left(\Delta \phi^{w-o}\right)$ for samples with aqueous phases containing $10 \mathrm{mM} \mathrm{LiCl}, \mathrm{RbCl}$, or $\mathrm{CsCl}$ in contact with the same organic phase. ${ }^{26}$

Impedance spectroscopy and capacitance analysis.- Impedance spectroscopy data are often used in ITIES studies to obtain the capacitance. The most commonly used equivalent circuit for interpretation of data is the Randles equivalent circuit shown in Fig. 6a. Only the low frequency data below $50 \mathrm{~Hz}$ are typically used for such interpretations ${ }^{50,51}$ and we followed the practice by utilizing the frequency range $0.2-40 \mathrm{~Hz} .{ }^{8,9,52}$ The data were fit to the following function:

$$
Z=R_{s}+\left(Z_{c}^{-1}+Z_{f}^{-1}\right)^{-1}
$$

where $Z$ is the measured impedance of the Randles equivalent circuit shown in Figure $6 \mathrm{a}, R_{s}$ is the solution resistance, $Z_{c}=(j \omega C)^{-1}$ is the capacitive impedance and $Z_{f}=Y_{0}^{-1}(j \omega)^{-1 / 2}$ is the Warburg impedance, where $\omega$ is the angular frequency, $j=\sqrt{-1}, Y_{0}$ is the faradaic admittance coefficient and $C$ is capacitance, which for this purpose is the interfacial differential capacitance. Figures $6 \mathrm{~b}$ and $6 \mathrm{c}$ show two representative fits to the impedance data from the $\mathrm{NaCl}$ sample at $\Delta \phi^{w-o}=-0.224$ and $0.246 \mathrm{~V}$. The non-linear least squares fit results for $R_{s}, C$, and $Y_{0}$ are listed in Table $\mathrm{S} 1$ in supplementary materials. The variation of differential capacitance with $\Delta \phi^{w-o}$ is shown in Figure 7. 

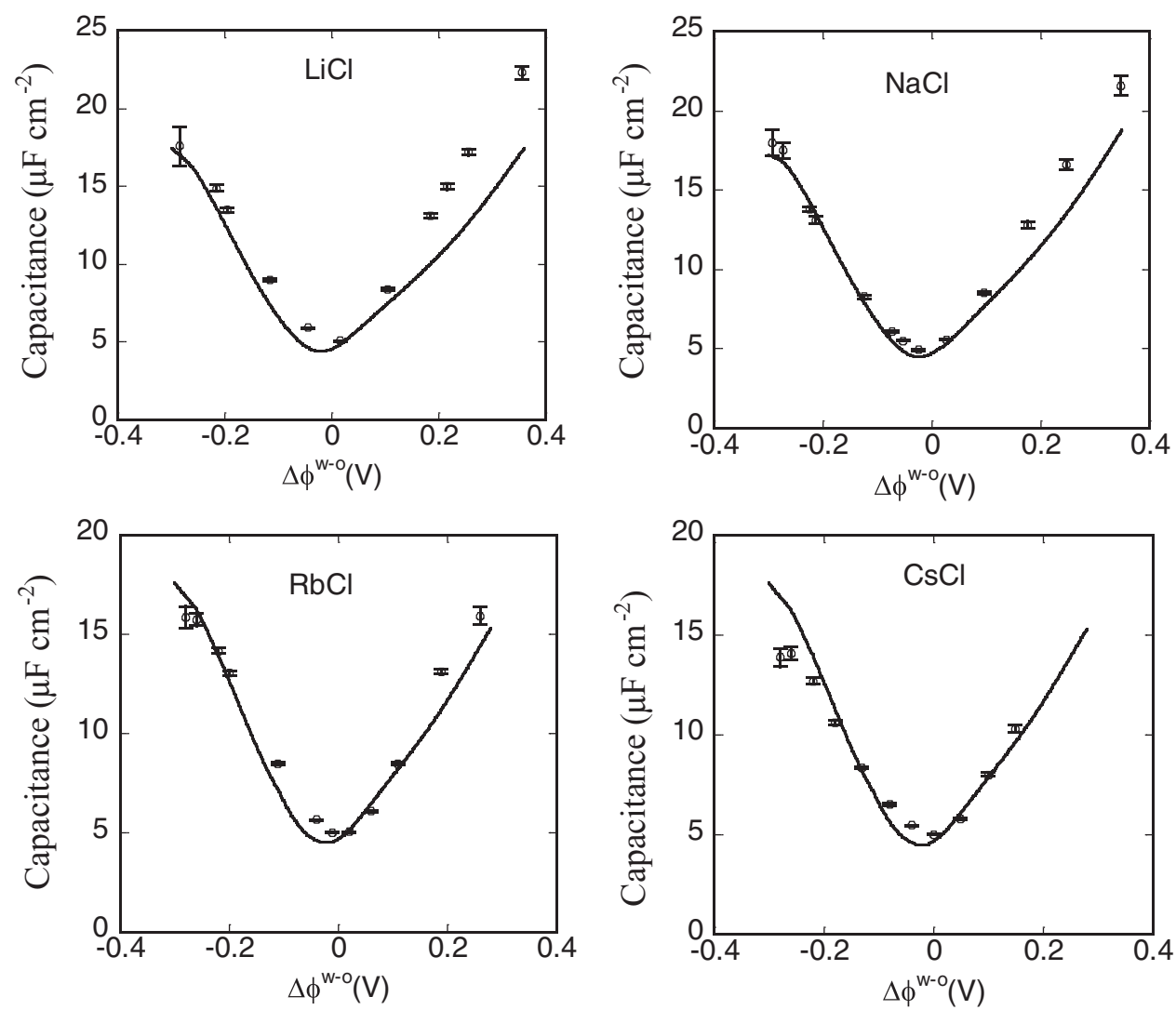

Figure 7. Differential capacitance vs. applied potential difference $\Delta \phi^{w-o}$ for liquid-liquid interfaces between $10 \mathrm{mM} \mathrm{XCl} \mathrm{(in} \mathrm{water)} \mathrm{and} 5 \mathrm{mM} \mathrm{BTPPATPFB} \mathrm{(in}$ 1, 2-dichloroethane), where $\mathrm{X}=\mathrm{Li}^{+}, \mathrm{Na}^{+}, \mathrm{Rb}^{+}$, and $\mathrm{Cs}^{+}$. Symbols represent differential capacitance data from impedance spectroscopy, solid lines are calculated from the PB-PMF theory.

Even though the non-linear least squares fit for the impedance data to the Randles circuit was good (Fig. 6), this does not mean that the differential capacitance parameter in the equivalent circuit represents the true differential capacitance of the sample interface. Nevertheless, we can test this because our X-ray reflectivity data probes the interfacial ion distribution on the nanoscale and the PBPMF analysis of these X-ray data yield ion distributions that can subsequently be used to calculate the differential capacitance. Values of the differential capacitance can then be compared with the results for the differential capacitance parameter from the Randles equivalent circuit analysis of the impedance spectroscopy. Before doing this, we make a few comments about the results for differential capacitance parameters from other equivalent circuit models of the interface.

A number of different equivalent circuits have been introduced to describe the behavior of ion distributions near the ITIES. ${ }^{7,8,18-20,53}$ As with the Randles equivalent circuit that we have used, these equivalent circuits often provide an acceptable fit of impedance spectroscopy data. First, we note that the simplest equivalent circuit, consisting of a capacitor and resistor in series (Fig. 6a, with $\mathrm{Z}_{\mathrm{f}}$ deleted), does not fit the data nearly as well as the Randles equivalent circuit, yet it yields a capacitance only about 5\% larger than the value from the Randles equivalent circuit model. An example of a more complicated equivalent circuit has been demonstrated in Ref. 53 for the purposes of fitting the high frequency semi-circular (or semi-elliptical in our case) part of the real and imaginary parts of the impedance, as well as accounting for parasitic couplings of the reference and counter electrodes. We had limited success fitting the high frequency part of our data, and could only fit the mid-frequency range $(40-3000 \mathrm{~Hz})$ of these data, most likely because of the larger size and different geometry of our sample cell compared to that used in Ref. 53. As stated in Ref. 53, the high frequency semicircle is a result of the stray capacitance and resistance associated with the parasitic coupling of the reference and counter electrodes, as well as the resistance of the bulk solution. None of these effects are the direct consequence of the double layer capacitance that is of interest in this study. In fact, fitting our data to the two-terminal equivalent circuit in Figure 6 of Ref. 53 which includes these effects, did not yield significantly different values for the interfacial differential capacitance.

The differential capacitance can also be calculated by differentiating the interfacial excess charge with respect to applied potential difference, $C=d Q_{t o t}\left(\Delta \phi^{w-o}\right) / d \Delta \phi^{w-o}$, where $Q_{t o t}\left(\Delta \phi^{w-o}\right)$ could be derived from either interfacial tension measurements or from the PBPMF analysis. The excellent agreement between these two methods of determining $Q_{t o t}\left(\Delta \phi^{w-o}\right)$, as previously shown in Fig. 5, indicates that the results will be equivalent, and Fig. 7 shows the differential capacitance calculated from the PB-PMF results. Although there is fair agreement in the region of negative $\Delta \phi^{w-o}$, values of the differential capacitance parameter from Randles equivalent circuit analysis of impedance spectroscopy do not match the differential capacitance determined by the PB-PMF calculation for positive $\Delta \phi^{w-o}$, especially for $\mathrm{LiCl}$ and $\mathrm{NaCl}$. The PB-PMF calculation also predicts the minimum in differential capacitance at slightly negative value of $\Delta \phi^{w-o}$, $-25 \mathrm{mV}$, most likely because the Gaussian minimum in Eq. 5 is taken to be invariant for all values of $\Delta \phi^{w-o}$. The Gaussian minimum biases the system toward $\mathrm{TPFB}^{-}$adsorption, even at $\Delta \phi^{w-o}=0$; therefore a slightly negative value of $\Delta \phi^{w-o}$ is required to produce a minimum in the capacitance calculated from PB-PMF theory. We have previously demonstrated that a different theoretical approach that accounts for ion-ion correlations through an excess chemical potential does not bias the system toward $\mathrm{TPFB}^{-}$adsorption at $\Delta \phi^{w-o}=0$ [28]; nevertheless, this approach does not improve the overall match between theoretical and experimental capacitance. In addition, our $\mathrm{X}$-ray experiments have not been able to distinguish between these two approaches. 
The differential capacitance determined by applying the Randles equivalent circuit to our impedance spectroscopy measurements is usually larger than values determined from the PB-PMF analysis (or, similar values from the interfacial tension measurements). For the two examples shown in Fig. 6 for $-0.224 \mathrm{~V}$ and $0.246 \mathrm{~V}$ the values are $14.2 \mu \mathrm{F} \mathrm{cm}^{-2}$ and $13.5 \mu \mathrm{F} \mathrm{cm}^{-2}$ from the PB-PMF theory compared to $13.8 \mu \mathrm{F} \mathrm{cm} \mathrm{cm}^{-2}$ and $16.6 \mu \mathrm{F} \mathrm{cm} \mathrm{cm}^{-2}$ from impedance spectroscopy measurements. Although the values at negative potentials are similar, the values from impedance spectroscopy at positive potentials are approximately $20 \%$ higher.

\section{Conclusions}

A Poisson-Boltzmann potential of mean force analysis (PB-PMF) that incorporates ion potentials of mean force to describe the role of liquid structure, molecular and ion correlations, as well as ion-specific effects, agrees well with $\mathrm{X}$-ray reflectivity data and interfacial tension measurements from the liquid-liquid interface between aqueous and organic electrolyte solutions. This demonstrates that a characterization of the ion distribution on the sub-nanometer scale, from X-ray reflectivity, is consistent with the results of a technique, interfacial tension, which probes the integrated ion distribution. However, the use of impedance spectroscopy to determine the differential capacitance of the interface is in only fair agreement with the PB-PMF analysis.

Samec and co-authors also reported limited agreement between interfacial tension and differential capacitance for the water/DCE electrolyte solution interface over a narrow range of potentials close to the zero charge potential. ${ }^{7,8,53}$ They proposed that the measured discrepancy between tension and impedance spectroscopy that results at larger potentials is an artifact arising from the inadequate representation of the interface and/or the electrochemical cell by the Randles equivalent circuit. Our results demonstrate that the PB-PMF model provides an excellent description of both nanoscale and macroscale characterizations of the interface by X-ray reflectivity and interfacial tension measurements. This understanding of the nanoscale ion distribution allowed us to show that the differential capacitance parameter in the Randles equivalent model, as determined by its application to impedance spectroscopy data, does not correctly describe the differential capacitance of liquid-liquid interfaces when the electric potential difference between the bulk electrolyte phases is larger than approximately $\pm 100 \mathrm{mV}$. Early studies that established the use of the Randles equivalent were generally carried out over a limited range of electric potential differences, within approximately $\pm 100 \mathrm{mV}$. However, the development of new supporting electrolytes for the organic phase has increased the range of the polarization window to $\pm 300 \mathrm{mV}$ or more. The results presented in this paper call for a re-evaluation of equivalent circuits for the analysis of impedance spectroscopy over this larger range of electric potential differences.

\section{Acknowledgments}

MLS and PV acknowledge support from NSF-CHE-0910825. Binhua Lin and Mati Meron are acknowledged for assistance with X-ray experiments at ChemMatCARS, Sector 15 of the Advanced Photon Source (APS), which is supported by NSF-CHE-1346572. The APS at Argonne National Laboratory is supported under DOE-BES Contract No. DE-AC02-06CH11357.

\section{References}

1. P. Vanysek, Electrochemistry on Liquid/Liquid Interfaces. Vol. 39, 1985, Berlin: Springer-Verlag.

2. Z. Samec, Electrochemistry at the Interface Between Two Immiscible Electrolyte Solutions, Pure Appl. Chem., 76(12), 2147 (2004).

3. H. H. Girault and D. H. Schiffrin, Electrochemistry of liquid-liquid interfaces, in Electroanalytical Chemistry, A. J. Bard, Editor, 1989, Marcel Dekker: New York.

4. H. H. Girault, Charge transfer across liquid-liquid interfaces, in Modern Aspects of Electrochemistry, J. O. M. Bockris, Editor, 1993, Plenum Press, New York.

5. A. G. Volkov, Liquid interfaces in chemistry and biology. 1998, New York: Wiley.

6. J. E. B. Randles, Kinetics of rapid electrode reactions, Discussion of the Faraday Society, 1, 11 (1947).
7. A. Trojanek et al., Limited agreement between the interfacial tension and differential capacity data for the polarised water|1,2-dichloroethane interface, Journal of Electroanalytical Chemistry, 565(2), 243 (2004).

8. Z. Samec, J. Langmaier, and A. Trojánek, Evaluation of parasitic elements contributing to experimental cell impedance: impedance measurements at interfaces between two immiscible electrolyte solutions, J. Chem. Soc., Faraday Transactions, 92, 3843 (1996).

9. C. M. Pereira et al., Differential capacitance of liquid/liquid interfaces: effect of electrolytes present in each phase, J. Chem. Soc. Faraday Trans., 90, 143 (1994)

10. H. H. Girault and D. H. Schiffrin, Thermodynamic surface excess of water and ionic solvation at the interface between immiscible liquids, J. Electroanal. Chem., 150, 43 (1983).

11. D. Homolka et al., The double layer at the interface between two immiscible electrolyte solutions: structure of the water/nitrobenzene interface, J. Electroanal. Chem., 159, 233 (1983).

12. C. Yufei et al., Interfacial capacitance and ionic association at electrified liquid/liquid interfaces, J. Chem. Soc. Faraday Trans., 87, 107 (1991).

13. C. M. Pereira et al., On the capacity of liquid-liquid interfaces, Chem. Phys. Lett., 268, 13 (1997).

14. C. M. Pereira et al., Ion association at liquid/liquid interfaces, J. Electroanal. Chem., 436, 9 (1997).

15. G. Gouy, Constitution of the electric charge at the surface of an electrolyte, $J$. Physique, 9, 457 (1910).

16. D. L. Chapman, A contribution to the theory of electrocapillarity, Phil. Mag. Ser, 25, 475 (1913).

17. Z. Samec, V. Marecek, and D. Homolka, The double layer at the interface between two immiscible electrolyte solutions. Part II. Structure of the water/nitrobenzene interface in the presence of 1:1 and 2:2 electrolytes, J. Electroanal. Chem., 187, 31 (1985).

18. A. Reindl and M. Bier, Impedance spectroscopy of ions at liquid-liquid interfaces, Physical Review E, 88, 052312 (2013).

19. H. N. Wang, A. Thiele, and L. Pilon, Simulations of cyclic voltammetry for electric double layers in asymmetric electrolytes: a generalized modified poisson-nernstplanck model, Journal of Physical Chemistry C, 117(36), 18286 (2013).

20. E. K. Lenzi et al., A connection between anomalous poisson-nernst-planck model and equivalent circuits with constant phase elements, Journal of Physical Chemistry $C, \mathbf{1 1 7}(45), 23685$ (2013)

21. T. Huber, O. Pecina, and W. Schmickler, The influence of the ions on the capacity of liquid | liquid interfaces, J. Electroanal. Chem., 467, 203 (1999).

22. L. I. Daikhin, A. A. Kornyshev, and M. Urbakh, Ion penetration into an 'unfriendly medium' and the double layer capacitance of the interface between two immiscible electrolytes, J. Electroanal. Chem., 500, 461 (2001).

23. L. I. Daikhin and M. Urbakh, Double layer capacitance and a microscopic structure of electrified liquid-liquid interfaces, J. Electroanal. Chem., 560, 59 (2003).

24. G. Luo et al., Ion distributions near a liquid-liquid interface, Science, 311, 216 (2006).

25. G. Luo et al., Ion distributions at the nitrobenzene-water interface electrified by a common ion, J. Electroanal. Chem., 593, 142 (2006).

26. B. Hou et al., Ion distributions at the water/1,2-dichloroethane interface: Potential of mean force approach to analyzing X-ray reflectivity and interfacial tension measurements, J. Phys. Chem. B, 117, 5365 (2013).

27. N. Laanait et al., Communications: monovalent ion condensation at the electrified liquid/liquid interface, J. Chem. Phys., 132, 171101-1 (2010).

28. N. Laanait et al., Tuning ion correlations at an electrified soft interface, Proc. Nat. Acad. Sci. (USA), 109, 20326 (2012).

29. N. Laanait, Ion correlations at electrified soft matter interfaces, in Department of Physics. 2011, University of Illinois at Chicago: Chicago.

30. H. M. Backes, E. Bender, and G. Maurer, Interfacial tensions in binary and ternary liquid-liquid systems, Chemical Engineering Science, 45(1), 12 (1990).

31. D. J. Fermín et al., Photoinduced electron transfer at liquid/liquid interfaces. 1. photocurrent measurements associated with heterogeneous quenching of zinc porphyrins, J. Phys. Chem. B, 102, 10334 (1998).

32. B. Hou, Ion distributions at electrified liquid-liquid interfaces: Microscopic and macroscopic measurements, in Department of Physics, 2011, University of Illinois at Chicago: Chicago.

33. G. Luo et al., Structure of the interface between two polar liquids: nitrobenzene and water, J. Phys. Chem. B, 110, 4527 (2006).

34. G. Luo et al., The width of the water/2-heptanone liquid-liquid interface, Electrochem. Comm., 7, 627 (2005).

35. D. M. Mitrinovic et al., Noncapillary-wave structure at the water-alkane interface, Phys. Rev. Lett., 85, 582 (2000).

36. A. Goebel and K. Lunkenheimer, Interfacial tension of the water/n-alkane interface, Langmuir, 13, 369 (1997).

37. A. H. Demond and A. S. Lindner, Estimation of interfacial tension between organic liquids and water, Environ. Sci. Technol., 27, 2318 (1993).

38. Z. Zhang et al., X-ray scattering from monolayers of $\mathrm{F}\left(\mathrm{CF}_{2}\right)_{10}\left(\mathrm{CH}_{2}\right)_{2} \mathrm{OH}$ at the water-(hexane solution) and water-vapor interfaces, J. Chem. Phys., 110, 7421 (1999).

39. P. S. Pershan and M. L. Schlossman, Liquid Surfaces and Interfaces: Synchrotron $X$-ray Methods, Cambridge University Press: Cambridge, 2012.

40. R. Kjellander and H. Greberg, Mechanisms behind concentration profiles illustrated by charge and concentration distributions around ions in double layers, J. Electroanal. Chem., 450, 233 (1998).

41. A. G. Volkov and D. W. Deamer, Liquid-Liquid Interfaces: Theory and Methods (Boca Raton, CRC, 1996).

42. D. Horinek and R. R. Netz, Specific Ion Adsorption at Hydrophobic Solid Surfaces, Phys. Rev. Lett., 99, 226104 (2007). 
43. V.P. Pawar, Dielectric relaxation of propan-1-ol with chlorobenzene, 1,2 dichloroethane, and dimethylene chloride at $(288,298,308$, and 318) $\mathrm{K}$ using timedomain reflectometry technique, J. Chem. Eng. Data, 51, 882 (2006).

44. D. M. Burley, V. C. L. Hutson, and C. W. Outhwaite, Calculation of the thermodynamic properties of electrolyte solutions using a modified Poisson-Boltzmann equation, Mol. Phys., 23(5), 867 (1972).

45. C. W. Outhwaite, L. B. Bhuiyan, and S. Levine, Theory of the electric double layer using a modified Poisson-Boltzmann equation, J. Chem. Soc. Faraday II, 76, 1388 (1980).

46. C. D. Wick and L. X. Dang, Molecular dynamics study of ion transfer and distribution at the interface of water and 1,2-dichloroethane, J. Phys. Chem. C, 112(3), 647 (2008).

47. F. P. Buff, R. A. Lovett, and F. H. Stillinger, Interfacial density profile for fluids in the critical region, Phys. Rev. Lett., 15, 621 (1965)
48. L. G. Parratt, Surface studies of solids by total reflection of X-rays, Phys. Rev., $\mathbf{9 5}$ 359 (1954).

49. G. Lippmann, Relations entre les phenoménes électriques et capillaires, Ann. Chim Phys., 5, 494 (1875).

50. J. R. Macdonald, Impedance spectroscopy, Annals of Biomedical Engineering, 20, 289 (1992).

51. Z. Samec et al., Interfacial Tension and Impedance Measurements of Interfaces between Two Immiscible Electrolyte Solutions, J. Electroanal. Chem., 483, 47 (2000).

52. M. C. Wiles et al., Experimental artifacts associated with impedance measurements at liquid-liquid interfaces, J. Electroanal. Chem., 278, 151 (1990).

53. A. Trojanek, V. Marecek, and Z. Samec, Some aspects of impedance measurements at the interface between two immiscible electrolyte solutions in the four-electrode cell, Electrochim. Acta, 2014 